\title{
Pictorial review of non-traumatic thoracic emergencies in the pediatric population
}

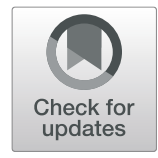

Mindy X. Wang ${ }^{1}$, Ameya Baxi ${ }^{2}$ and Dhanashree Rajderkar ${ }^{1 *}$

\begin{abstract}
Background: Non-traumatic thoracic emergencies are commonly encountered emergencies in children. These require prompt diagnosis in order to establish timely management, especially since these patients can rapidly decompensate with hemodynamic and respiratory failure.

Main text: The objective of this article is to review various non-traumatic emergent chest pathologies in the pediatric population that a radiologist can encounter in a day-to-day practice. These thoracic emergencies are divided into pleuroparenchymal, cardiac, vascular, esophageal, mediastinal, and thoracic cage etiologies. Conclusions: The timely diagnosis of these conditions is essential for the acute management of these emergencies.
\end{abstract}

Keywords: Thoracic emergencies, Pediatric, Non-traumatic, Imaging

\section{Background}

Non-traumatic thoracic emergencies are commonly encountered emergencies in children [1]. The clinical presentation of these emergencies are nonspecific, and many of the pathologies have similar clinical presentations. Common symptoms include stridor, cough, wheezing, respiratory distress, tachypnea, nasal flaring, and intercostal retractions. Swallowing problems, hemoptysis, fever, sepsis, chest pain, and failure to thrive may also be encountered. These emergencies require prompt diagnosis in order to establish timely management, especially since children can rapidly decompensate with hemodynamic and respiratory failure [1].

Radiology plays a critical role in the diagnosis and evaluation of these pathologies, in addition to monitoring their treatment response. The appropriate initial imaging method for acute respiratory failure is plain radiography, which is often sufficient for most cases; it is an affordable and easily available imaging modality. Ultrasound may be preferred to evaluate diaphragmatic motion and to diagnose pleural and pericardial effusions. Cross-sectional imaging, such as computed tomography (CT), may be used to evaluate pulmonary parenchymal

\footnotetext{
*Correspondence: rajdda@radiology.ufl.edu

${ }^{1}$ Department of Radiology, University of Florida College of Medicine, 1600

SW Archer Road, Gainesville, FL 32611, USA

Full list of author information is available at the end of the article
}

and mediastinal masses. Magnetic resonance imaging (MRI) is typically used for evaluating mediastinum and cardiovascular pathologies. These advanced imaging techniques also help elucidate relevant vascular and anatomical information. It is important to use the ALARA (as low as reasonably achievable) and Image Gently principles when imaging the pediatric population [1].

The objective of this article is to review various nontraumatic emergent chest pathologies that a radiologist can encounter in a day-to-day practice. We divide thoracic emergencies into six categories: (1) pleuroparenchymal, (2) cardiac, (3) vascular, (4) esophageal, (5) mediastinal, and (6) thoracic cage. Images presented in this article span the spectrum of emergent chest conditions encountered from day 0 of life to 18 years of age.

\section{Pleuroparenchymal etiologies Transient tachypnea of the newborn}

Transient tachypnea of the newborn occurs as a result of pulmonary edema from delayed clearance of fetal alveolar fluid [2]. This presents commonly in infants born by cesarean section, and it can occur in the infants born to the mothers with diabetes, asthma, prolonged labor, and fetal distress [2]. This is a diagnosis of exclusion and usually self resolves within 3 days. The most common findings on radiograph are interstitial edema and pleural effusions. However, other radiographic features include 


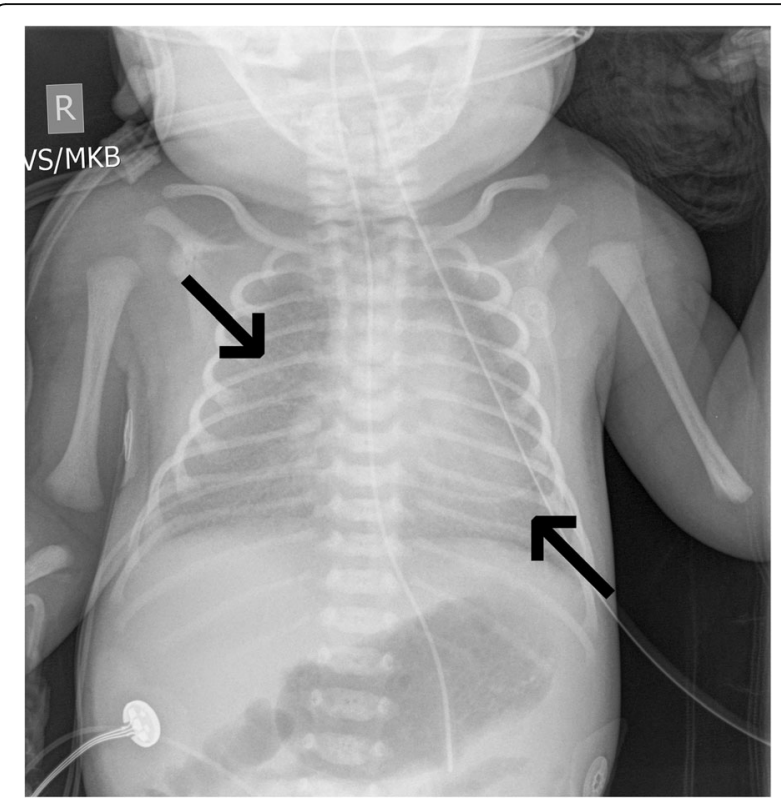

Fig. 1 A 0-day-old male with bilateral interstitial pulmonary haziness (black arrows) suggestive of transient tachypnea of the newborn

increased lung volumes, perihilar congestion, increased interstitial markings (Fig. 1), and fluid in the fissures [2]. These findings usually resolve $48 \mathrm{~h}$ after the birth.

\section{Pneumonia and related complications}

Pneumonia is the leading cause of death worldwide with 1.2 million deaths per year in children less than 5 years of age [3]. Early diagnosis and appropriate treatment is essential. In general, the most common cause is infections; however, the infectious organisms involved depend on the patient's age. In neonates ( 0 to 3 months of age), maternal flora, including group $\mathrm{B}$ streptococcus and gram-negative bacteria, are common causes. In children older than 1 week, Streptococcus pneumoniae is the most common bacterial cause. In children ages 3 months to 5 years, over half of cases are related to viral respiratory infections. In children age 5 years or older, atypical organisms, including Mycoplasma pneumoniae and Chymydophila pneumoniae, are more prevalent [3]. If the pneumonia is inadequately treated, it can infrequently progress to necrotizing pneumonia, lung abscess (Figs. 2a, b and 3), pneumatocele (Fig. 4), and empyemas. Reactive pleural effusions are relatively common. Relatively uncommon complications include bronchopleural fistula.

Routine chest radiography may aid the diagnosis of pneumonia (Fig. 5); however, the absence of radiographic findings does not rule out pneumonia as the findings can be delayed in presentation. Additionally, the radiographic findings are nonspecific and include well-defined to ill-defined airspace opacities, ground-glass opacities, prominent interstitial markings, pleural effusion, and lymphadenopathy.

Viral infections can appear as bilateral perihilar linear opacities, peribronchial markings, hyperinflation, and air trapping [1]. Bacterial infections usually present unilaterally with lobar opacities [1]. Round pneumonia is seen only in children less than age 8. Exudate that is unable to pass through pores of Kohn remains trapped in alveoli; this is seen as a round mass in radiography (Figs. 6a, b). Pulmonary hemorrhage can be indistinguishable from pneumonia. Occasionally, the hyperdense opacities might be suggestive of pulmonary hemorrhage in a correct clinical setting. These findings should be correlated clinically along with laboratory values whenever possible.

\section{Hypoplastic lungs}

Hypoplastic lungs is defined as deficient or incomplete development of the lungs [4]. Intra- or extra-thoracic abnormalities that interfere with normal lung development can lead to underdeveloped bronchi and alveoli [4]. There may be an absence of one or multiple lung lobes, and the degree of hypoplasia depends on the volume and duration of the cause. Infants with a history of oligohydramnios, which can occur secondary to fetal genitourinary abnormalities or prolonged rupture of membranes [5], are at high risk of the global
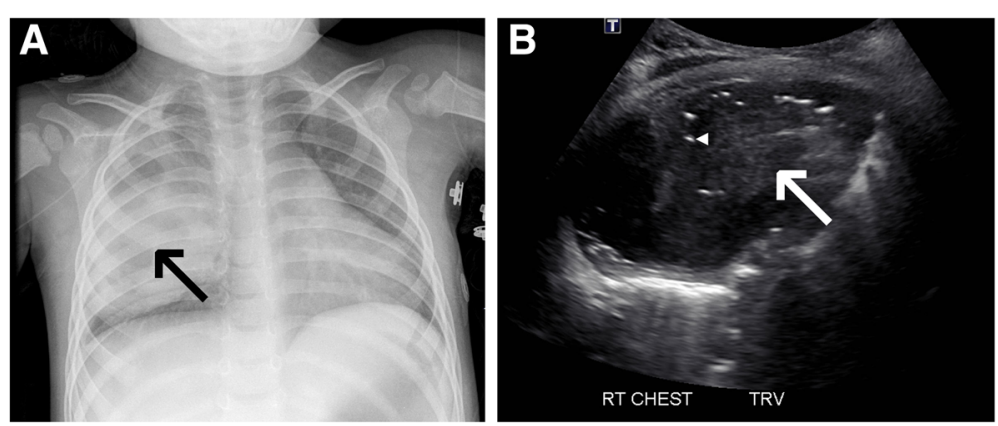

Fig. 2 a, b. Radiograph demonstrating air fluid level (black arrow) in the right lung field. Ultrasound showing a large abscess (white arrow) with air bubbles (white arrowhead) 


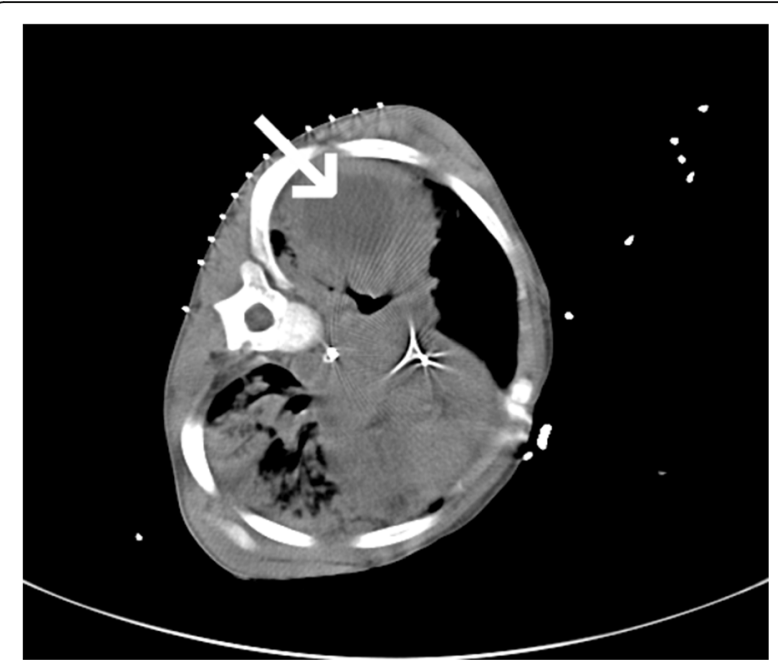

Fig. 3 A 4-year-old female with a 4 by 4-cm complicated fluid collection (white arrow) in the right lower lobe representing a lung abscess on CT

underdevelopment (Fig. 7). The causes of lung hypoplasia are congenital diaphragmatic hernia, extralobar sequestration, diaphragm agenesis, large pleural effusions, and asphyxiating thoracic dystrophy (Jenune syndrome) [4]. If hypoplasia is focal, radiographs reveal decreased aeration of the affected hemithorax (often in the right lung) (Fig. 8) and a small thoracic cage [4]. Absence of a single lobe/lung is usually well-compensated from birth. Pneumothorax occurs spontaneously as a serious complication due to over mechanical ventilation in a newborn (Fig. 9). Approximately, 35\% of the newborn infants who have spontaneous pneumothorax are diagnosed with renal pathology. Findings on radiographs include displacement of the mediastinum to the side with hypoplastic lung [4] and lack of lung markings peripheral to the visceral pleural edge. If fetal $M R$ is performed, it can assess the lung volumes [6].

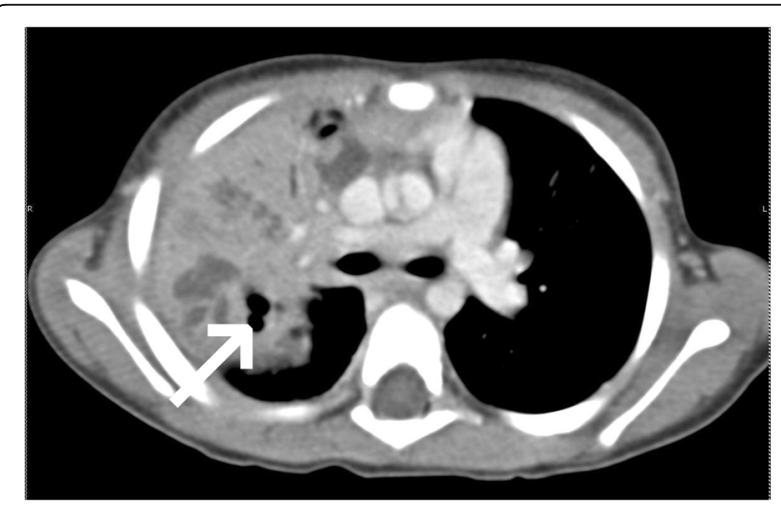

Fig. $4 \mathrm{CT}$ with contrast showing pneumatocele formation (white arrow)

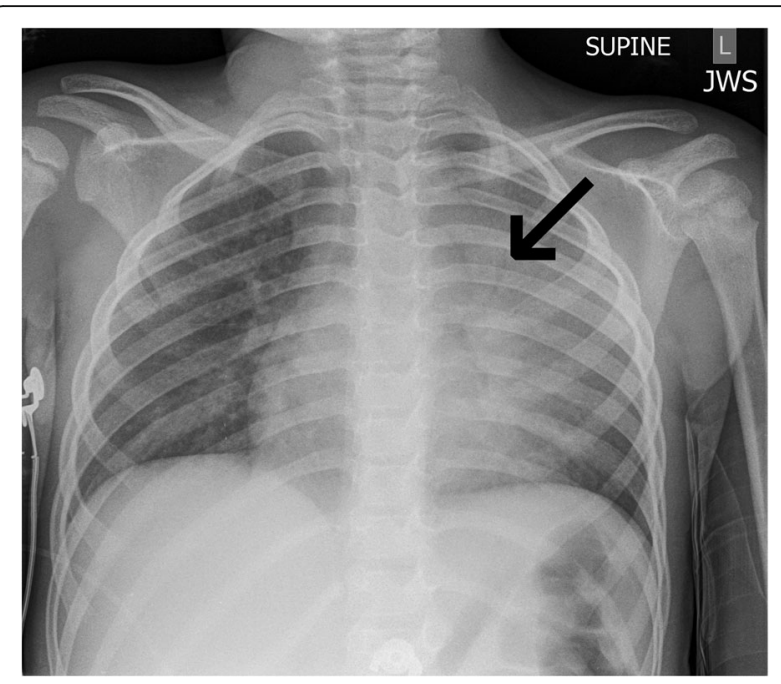

Fig. 5 A 3-year-old female with sickle cell disease and left upper lobe pneumonia (black arrow)

\section{Meconium aspiration syndrome}

Meconium aspiration syndrome (MAS) is respiratory distress in an infant secondary to intrapartum or intrauterine aspiration of meconium-stained amniotic fluid. Infants can present with mild to life-threatening respiratory failure. There are 25,000 to 30,000 cases that occur annually [7]. In utero hypoxia can lead to colonic activity and passage of meconium, which can result in meconium aspiration. This aspiration can cause mechanical obstruction, leading to air trapping, uneven ventilation, air leaks, hypoxemia, acidosis, and primary pulmonary hypertension [7]. Similarly, chemical inflammation and surfactant inactivation ultimately leads to the same presentation through atelectasis and intrapulmonary shunting [7]. Plain radiographs can demonstrate bilateral coarse opacities, areas of atelectasis alternating with areas of overinflation, or pneumothorax secondary to air-leak phenomenon (Fig. 10a, b) [8]. However, radiographs may be indistinguishable from neonatal pneumonia or pneumonia superimposed in MAS. Unless there is definite risk for infection in the perinatal period, prophylactic use of antibiotics in MAS is not recommended.

\section{Respiratory distress syndrome}

Respiratory distress syndrome (RDS), also known as hyaline membrane disease, results from surfactant deficiency in neonates. These neonates typically have a deficiency of mature type II pneumocytes. Risk factors for RDS include infants of mothers with poorly controlled diabetes, fetal asphyxia, maternal or fetal hemorrhage, and multiple gestations. On chest radiograph, there is hypoaeration from underinflation (Fig. 11), symmetric ground-glass opacification, effacement of normal pulmonary vessels, and prominent air bronchograms. 


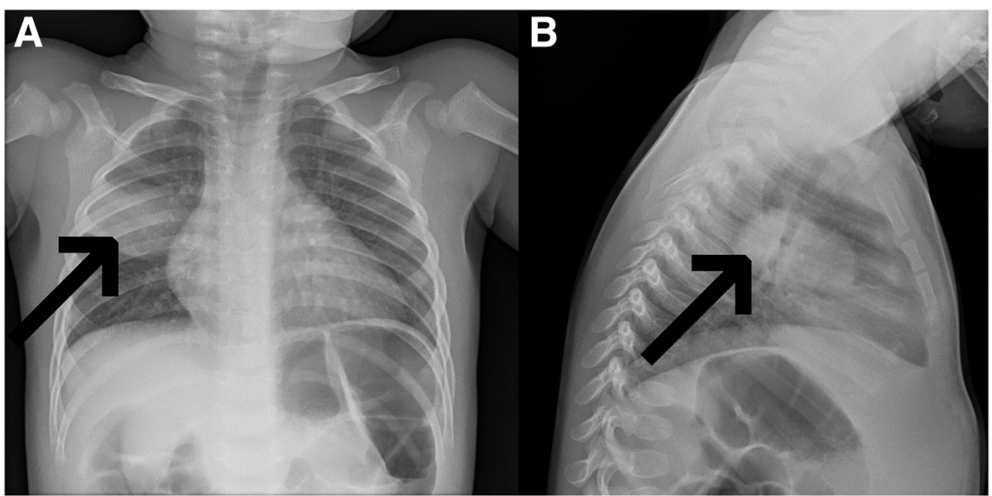

Fig. 6 a, b A 16-month-old male with well-defined round parenchymal opacity (black arrows) in the right mid-lung field on frontal and lateral films representing round pneumonia.

These findings can be seen shortly after birth. In severe cases, dense bilateral symmetric lung consolidation or "white-out" can be visualized [9]. Pulmonary hemorrhage is a rare complication that can occur following surfactant treatment. On radiograph, this can be seen as sudden dense airspace consolidation [9].

\section{Pulmonary interstitial emphysema}

Acute pulmonary interstitial emphysema (PIE) results from ruptured intra-alveolar air that extends into the interstitial pulmonary tissue. This is a complication of barotrauma in mechanically ventilated and extremely low birth weight newborns with surfactant deficiency disease who have received surfactant and high pressure ventilatory support [10]. This presentation serves as a warning sign for potential pneumothorax or pneumomediastinum. Imaging findings on chest radiography include linear and round bubble-like radiolucencies along the interstitium, which can be focal/diffuse or unilateral/ bilateral (Fig. 12) [10]. Diagnosis prompts lowering of

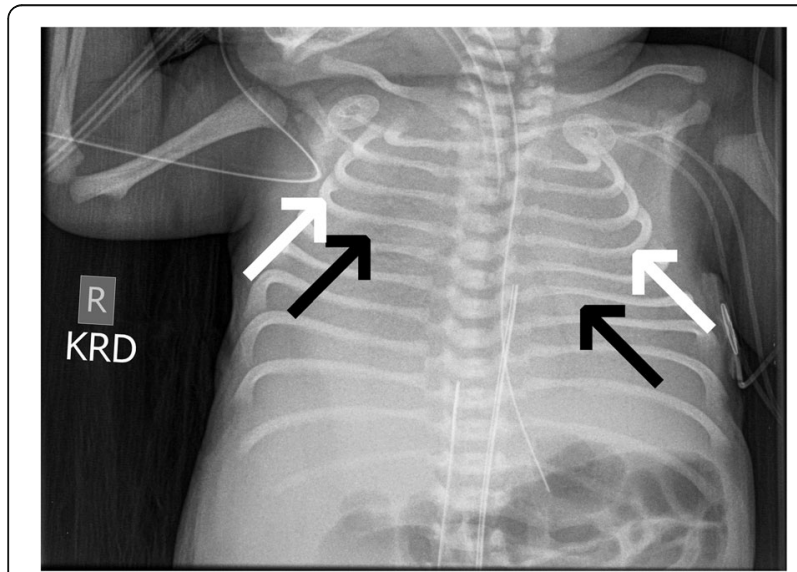

Fig. 7 A 1-day-old male with a history of Potter syndrome with hypoplastic lungs, bell-shaped thorax (white arrows), and airspace opacities likely reflecting diffuse alveolar atelectasis (black arrows) the ventilatory settings and prevention of the aforementioned complications. Acute PIE is usually transient.

\section{Congenital lobar emphysema}

Congenital lobar emphysema (CLE) is a developmental anomaly in which there is hyperinflation of one or more pulmonary lobes [11]. Airway obstruction can lead to a "check-valve" mechanism [1]. There are intrinsic causes of obstruction, such as bronchomalacia, meconium plugs, and mucosal webs; and extrinsic causes of obstruction, such as vascular anomalies, intrathoracic masses, and cysts [11]. Chest radiography demonstrates hyperlucent and overexpanded lobe with a left upper lobe and right middle lobe predilection [11]; however, any lobe can be affected [12]. The hyperinflated lungs can cause a mass effect, resulting in atelectasis and mediastinal shift (Figs. 13 and 14a, b) [12]. The management of CLE includes bronchoscopy to exclude endobronchial lesions and surgical resection of the affected lobe.

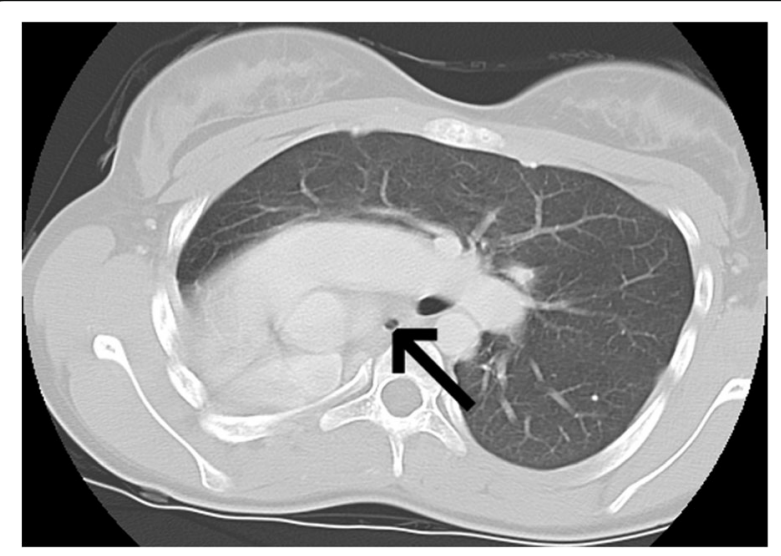

Fig. 8 A 13-year-old female with right lung agenesis (black arrow to small right bronchus) on axial $\mathrm{CT}$ 


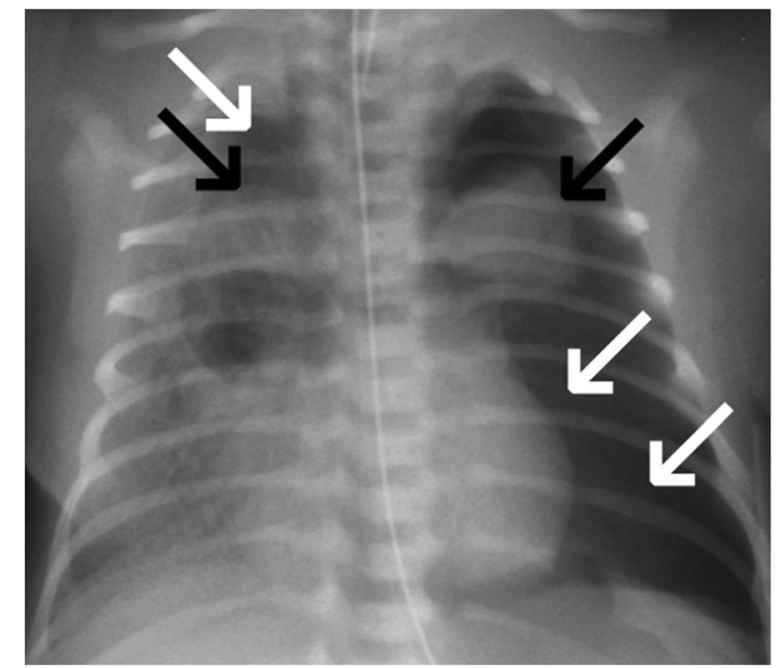

Fig. 9 A 0-year old male with bilateral pneumothoraces (white arrows) and very hypoplastic lungs (black arrows)

\section{Congenital diaphragmatic hernia}

Congenital diaphragmatic hernia $(\mathrm{CDH})$ is a lifethreatening developmental defect of the diaphragm with herniation of abdominal contents into the chest. Pulmonary hypoplasia is a complication of CDH. Posterior or Bochdalek hernia is the most common type [13], in which herniated contents include part of the liver, small and large bowel, stomach, and spleen. Herniation of the liver is predictive of the overall outcome. Right-sided herniation is associated with liver herniation. Bilateral hernias have been reported but are rare. $\mathrm{CDH}$ is diagnosed prenatally on obstetric ultrasound and then evaluated with fetal MRI. Initial chest radiography can show opacified hemithorax with mass effect and contralateral shift of the mediastinum (Figs. 15 and 16) [1]. There may be lucencies in the chest from herniated bowel loops, intrathoracic nasogastric tube,

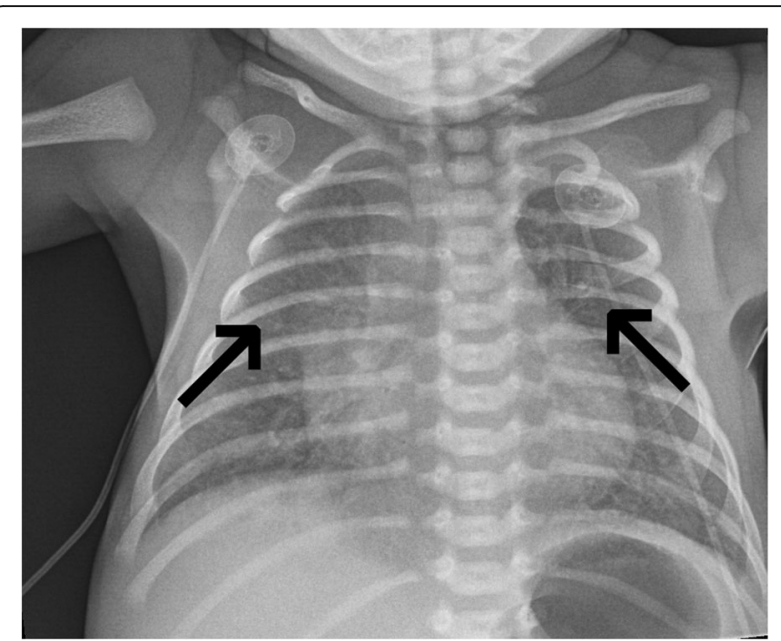

Fig. 11 A 33-week-old with hypoinflated lungs and mild diffuse haziness of the lungs bilaterally (black arrows) from respiratory distress syndrome

and altered course of an umbilical venous catheter. The management includes supportive therapy until hernia repair, which is usually done within a week, and immediate gastric decompression after birth. In the event of late presentation, the defect may lead to pulmonary hypoplasia on the affected side, in addition to pulmonary hypertension and the need for extra-corporeal membrane oxygenation [1].

\section{Asthma}

Asthma is an inflammatory lung disease with increased airway reactivity to various stimuli and at least partially reversible airflow obstruction. It is identified with the criteria of symptoms of recurrent coughing/wheezing, other causes excluded, risk factors of asthma, improvement with asthma therapy, and confirmatory physical examination and/or diagnostic

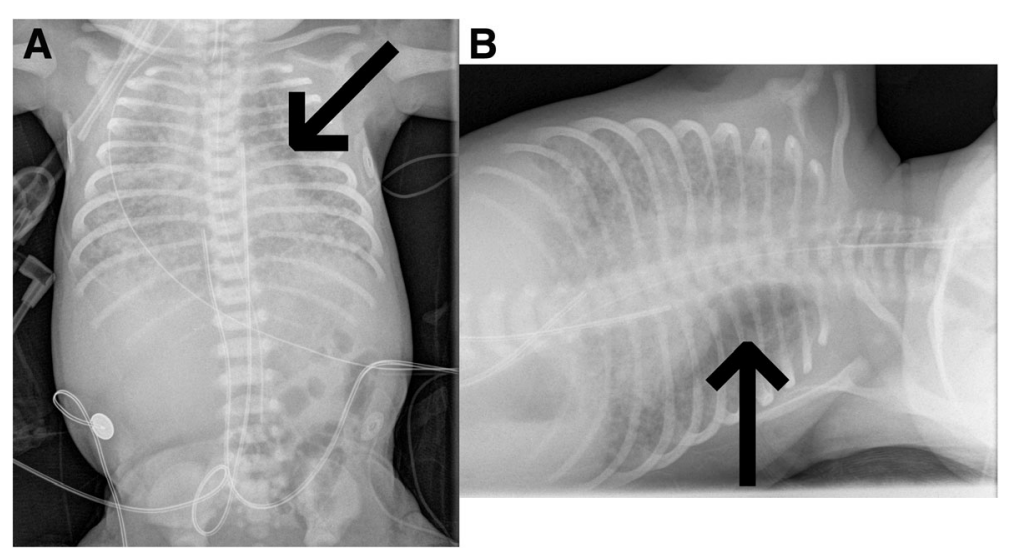

Fig. 10 a, b A 0-day-old male with severe meconium aspiration syndrome with lucency adjacent to the left cardiac and mediastinal borders (black arrows) on upright and decubitus films that represents a medial left pneumothorax 


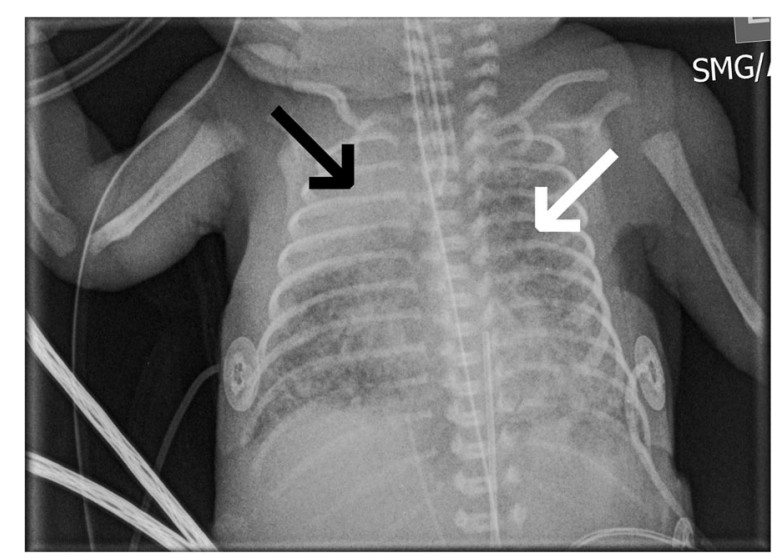

Fig. 12 A premature 9-day-old male with right upper lobe atelectasis (black arrow) and pulmonary interstitial emphysema (white arrow)

tests [14]. Because infants have smaller caliber of airways, they experience more severe symptoms compared to those in older age groups [1]. Chest radiographs are indicated for complicated asthma. Chest radiograph findings that are suggestive of lower airway obstruction defect include increased peribronchial markings/peribronchial cuffing and increased air volume/hyperinflation bilaterally [15]. These findings are more prevalent in patients younger than 5 years of age with persistent cough and can aid in the diagnosis of asthma, especially in the setting where these patients cannot perform spirometry [15]. One retrospective study found that children diagnosed

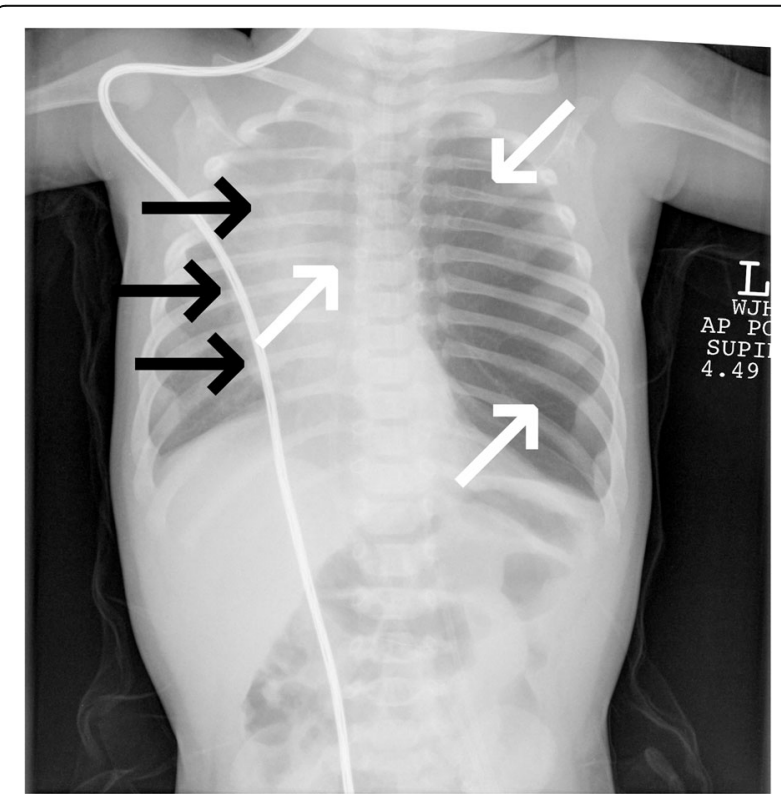

Fig. 13 A 82-day-old male with lucency (white arrows) in the left hemithorax causing mass effect on the mediastinum (black arrows), which is suggestive of congenital lobar emphysema
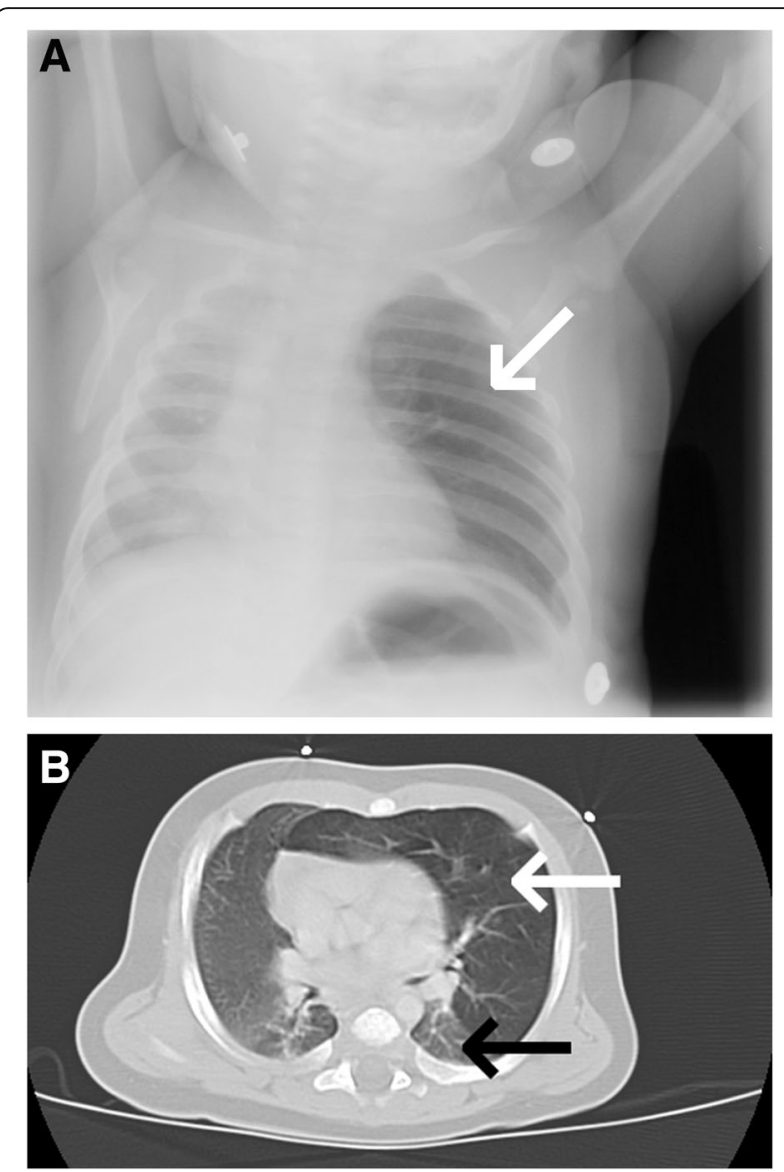

Fig. 14 a, b A 86-day-old male with hyperexpanded left lung (white arrow) on radiograph and axial $\mathrm{CT}$ in contrast to the normal left lower lobe (black arrow)

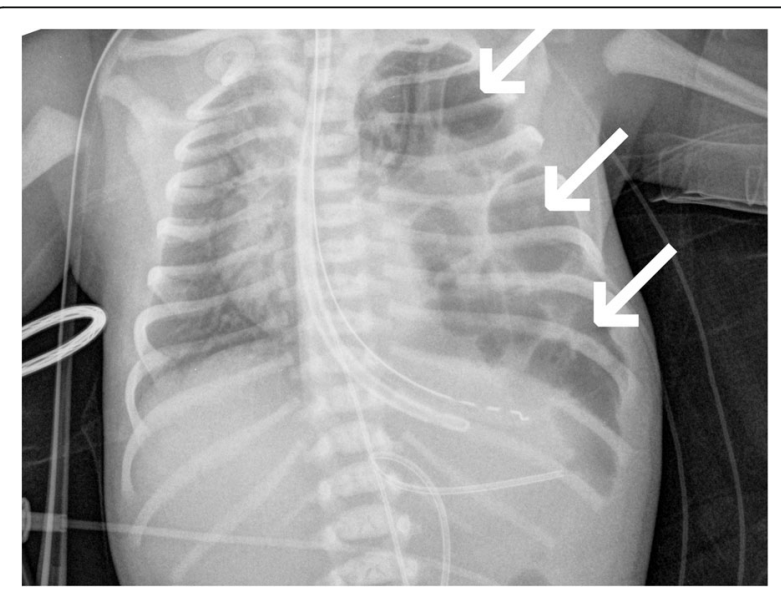

Fig. 15 A 1-day-old male with lucencies in the left hemothorax (white arrows) with associated mediastinal shift suggesting left sided congenital diaphragmatic hernia 


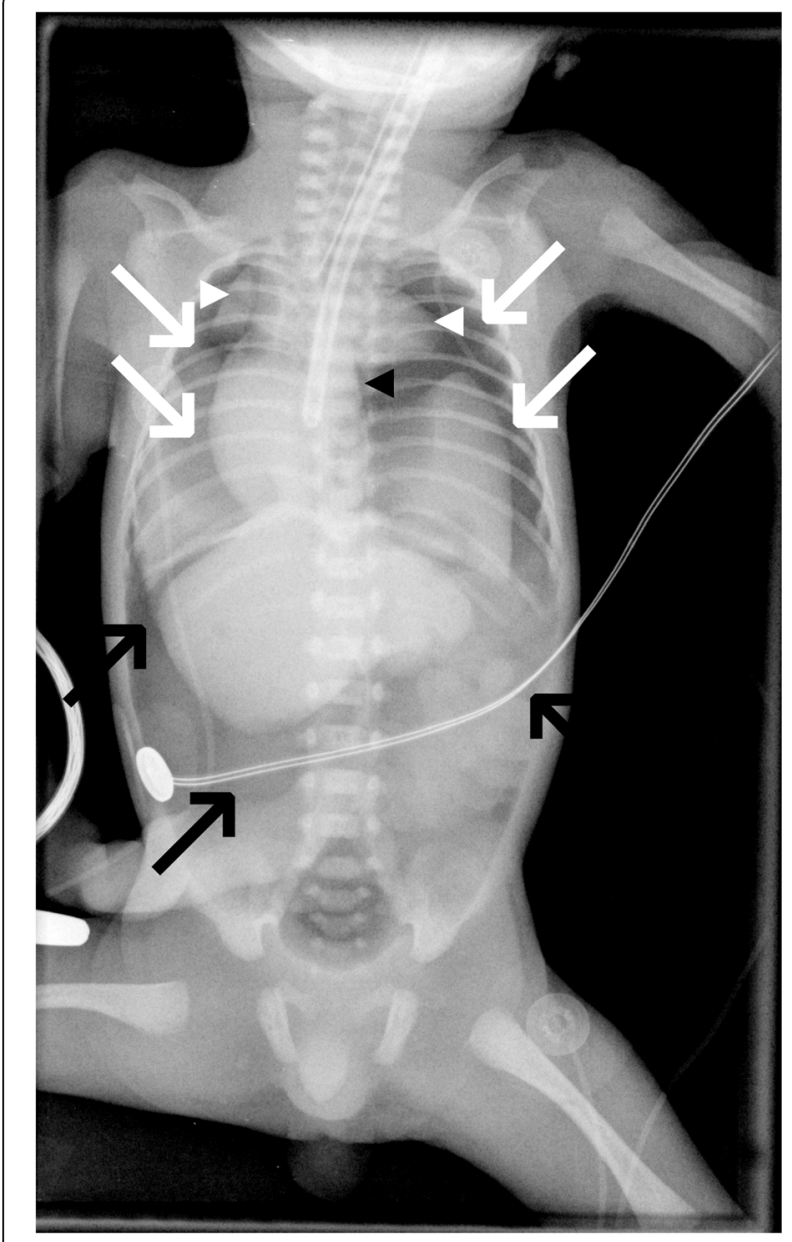

Fig. 16 A 0-year-old male with congenital diaphragmatic hernia with severe air leak. There is pneumomediastinum (black arrowhead) and pneumothoraces (white arrows) around each collapsed lung (white arrowheads). A large pneumoperitoneum is also present (black arrows)

with cough-variant asthma had chest radiographic findings suggestive of lower airway obstruction defect $30 \%$ of the time [15]. Superimposed pneumonia is a known complication (Fig. 17).

\section{Cardiac etiologies}

\section{Multivalvular large septal defects}

Congenital septal defects are the most common major congenital anomaly and include ventricular septal defects (VSD) and atrial septal defects (ASD). These defects are often diagnosed by fetal echocardiogram [16]. Radiograph may be nonspecific, but can support findings on echocardiogram.

VSD account for up to $40 \%$ of congenital cardiac malformations, which present early in life. Echocardiogram may be normal if the VSD is small; however, left-ventricular hypertrophy due to volume loading or right ventricular hypertrophy due to pulmonary hypertension or

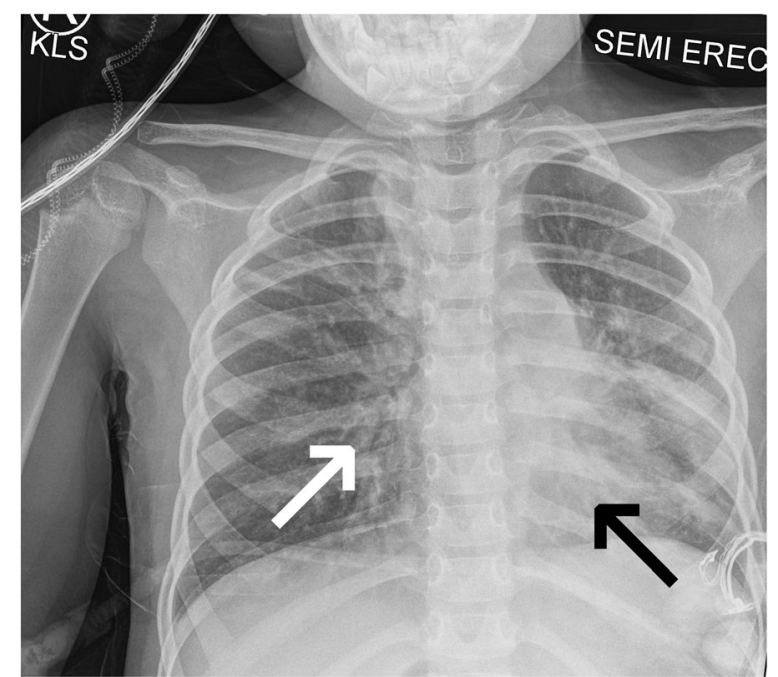

Fig. 17 A 3-year-old female with extensive peribronchial thickening from reactive airway disease (white arrow) complicated with lower lobe pneumonia (black arrow)

obstruction may be present [17]. Color flow Doppler is utilized to visualize the defect. On chest radiograph, engorged pulmonary vasculature and posteriorly deviated esophagus due to a dilated left atrium may be visualized [18]. Reformatted CT scans, catheter cardioangiography, and MR imaging may elucidate the typical appearances of congenital septal defects (Fig. 18). Based on these images, VSD can be classified by their

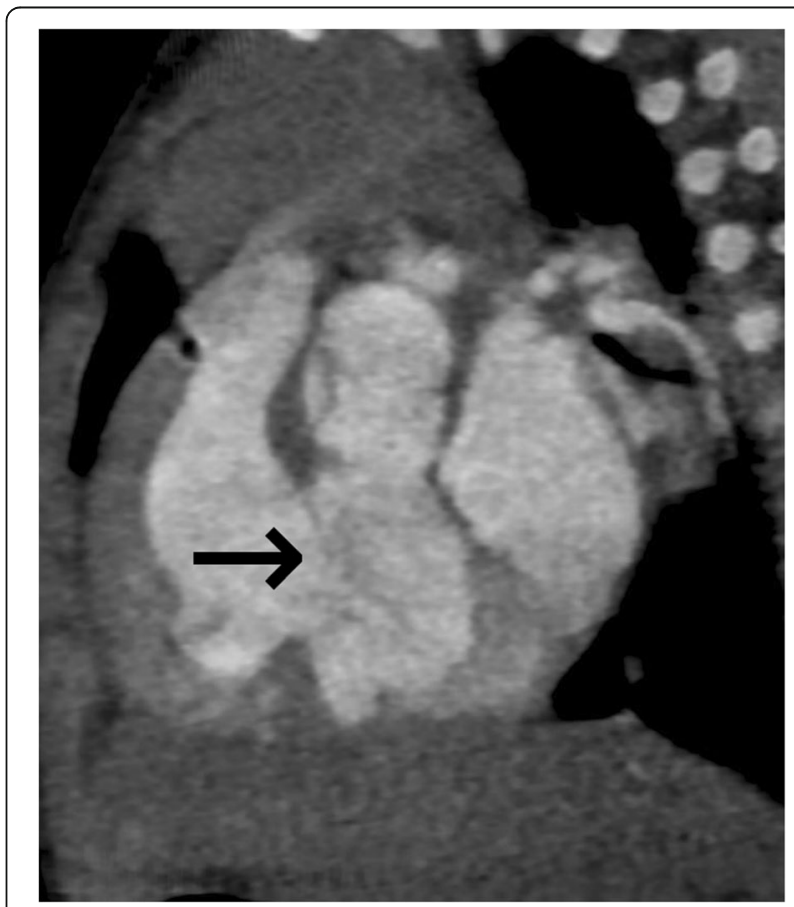

Fig. 18 A 5-month-old male with VSD (black arrow) seen on CT reconstruction 


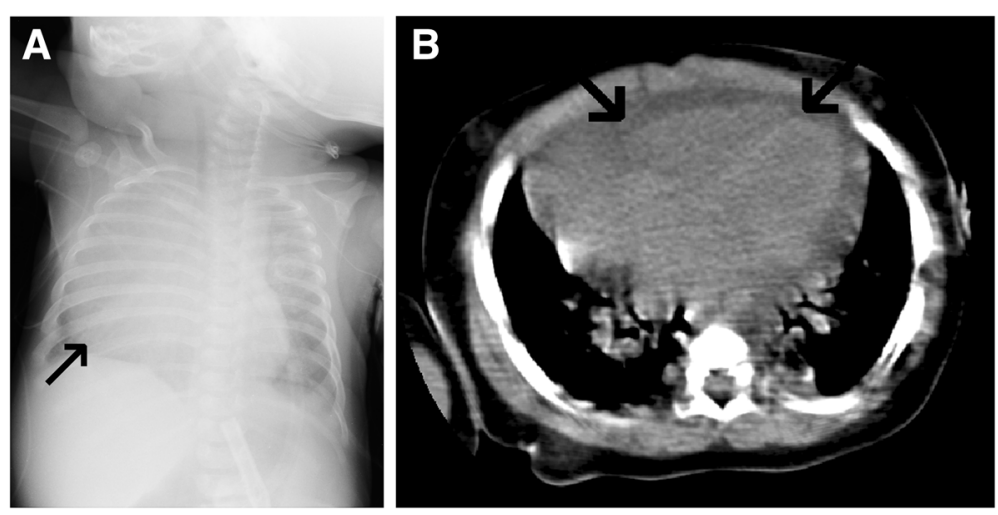

Fig. 19 a, b A 35-day-old female with pericardial effusion (black arrows) on radiograph and axial CT

appearance from the lumen of the right ventricle as perimembranous, muscular, or doubly committed juxta-arterial defects [19].

ASD is a persistent interatrial communication and occurs in various forms including ostium secundum, ostium primum, sinus venosus, and coronary sinus defects. The prevalence of ASDs is 3.89 per 1000 children. A large ASD can present early in life as an emergency. On chest radiograph, there will be prominent vascular markings. If the ASD is large, an enlarged cardiac silhouette may be visualized due to the fullness of the right atrium in addition to fullness of the anterior aspect of the cardiac silhouette due to dilated right ventricle [18]. Crosssectional imaging, including multidetector CT (MDCT) and cardiac MRI, are used in select cases before surgical repair or to evaluate associated complex congenital diseases.

\section{Pericardial effusion}

Pericardial effusion is an isolated finding or manifestation of systemic or cardiac disease. There is a wide clinical spectrum ranging from asymptomatic to cardiac

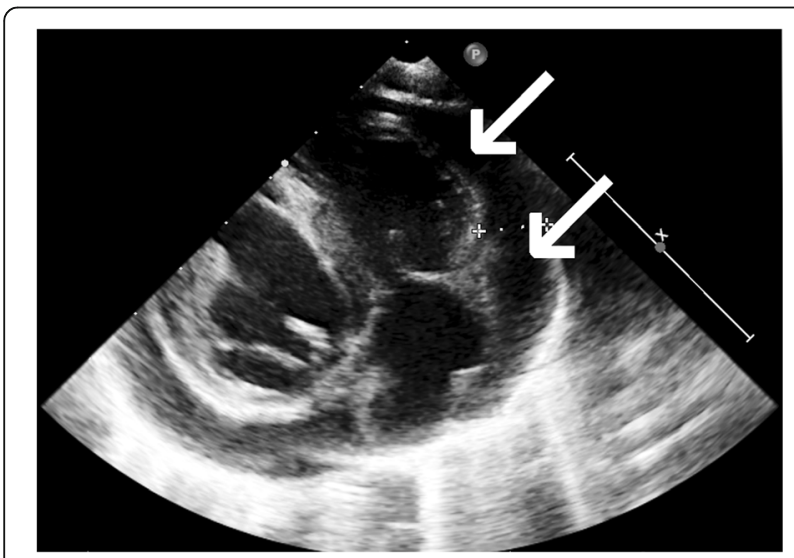

Fig. 20 A 6-month-old male with pericardial effusion (white arrows) on echocardiogram tamponade. There are various causes of this pathology. Infectious etiologies can be of viral origin (echovirus, Coxsackievirus, Parvovirus B19, Human herpesvirus 6, HIV), bacterial origin (tuberculous, Staphylococcus aureus, Haemophilus influenzae), and fungal origin in immunosuppressed patients (Aspergillosis, Candida, Blastomycosis) [20]. Noninfectious causes include autoimmune disease (juvenile idiopathic arthritis, systemic lupus erythematosus), uremic pericarditis, drug-induced (hydralazine, isoniazid, procainamide, chemotherapy), and pericardial metastasis from solid and hematologic malignancies [20]. On chest radiograph, significant pericardial effusion may demonstrate a triangular-shaped heart with smooth border or "water-bottle heart" (Fig. 19a) [21]. Additionally, lateral chest radiograph may demonstrate the Oreo cookie sign, where the pericardial fluid displaces the vertical lucent line behind the sternum (epicardial fat) anteriorly from a lucent vertical line (pericardial fat) posteriorly. The subcarinal angle may be widened as well. CT would demonstrate fluid around the myocardium (Fig. 19b). On echocardiography, it appears as an echo-free space between the two layers of pericardium (Fig. 20)

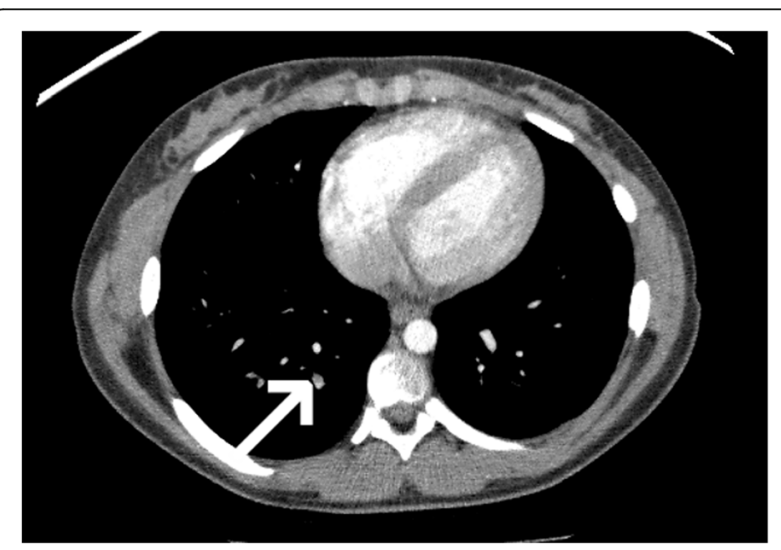

Fig. 21 A 13-year-old female with filling defect (white arrow) on axial CT indicating pulmonary embolism 


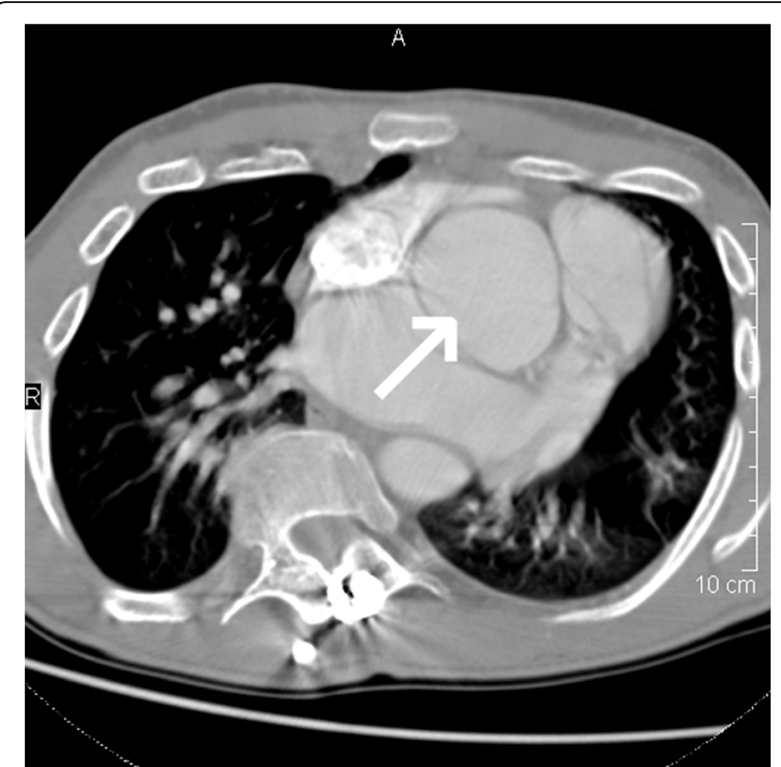

Fig. 22 A 25-year-old male with an aortic root aneurysm (white arrow) in the setting of Marfan's syndrome

[22]. However, it may be difficult to properly distinguish the etiology of the effusion just by echocardiogram. For example, intrapericardial fibrin and pyogenic exudates appear as loculations, and echogenicities are associated with complex exudates and hemopericardium. Transudates usually appear with more echo-free signal [22]. Cross-sectional imaging like MDCT and cardiac MRI can be used in select cases.

\section{Vascular etiologies}

\section{Pulmonary embolism}

Pulmonary embolism (PE) has been considered to be a rare occurrence in the pediatric population, but the exact incidence is unknown. However, the overall incidence is likely underestimated. One retrospective review of 3600 pediatric autopsies found a 3.7\% incidence of massive PE [23]. Risk factors vary depending on the age. In neonates, risk factors include dehydration, septicemia, peripartum asphyxia, and central venous catheter with associated thrombus [24]. Imaging serves as the primary diagnostic tool for PE, as the clinical presentation can be vague and other diagnostic tests lack specificity. Chest radiography is primarily helpful in excluding other lung pathologies [24]. Findings suggestive of PE on imaging include cardiac enlargement, pleural effusion, atelectasis, and parenchymal opacity. Other signs include the classic Westermark sign (peripheral oligemia), the Hampton hump (dome-shaped pleural based opacity), and Fleischner sign (dilated central pulmonary artery).

Ultrasound of the lower extremity can be utilized to find the thrombotic source. Ultrasound findings include

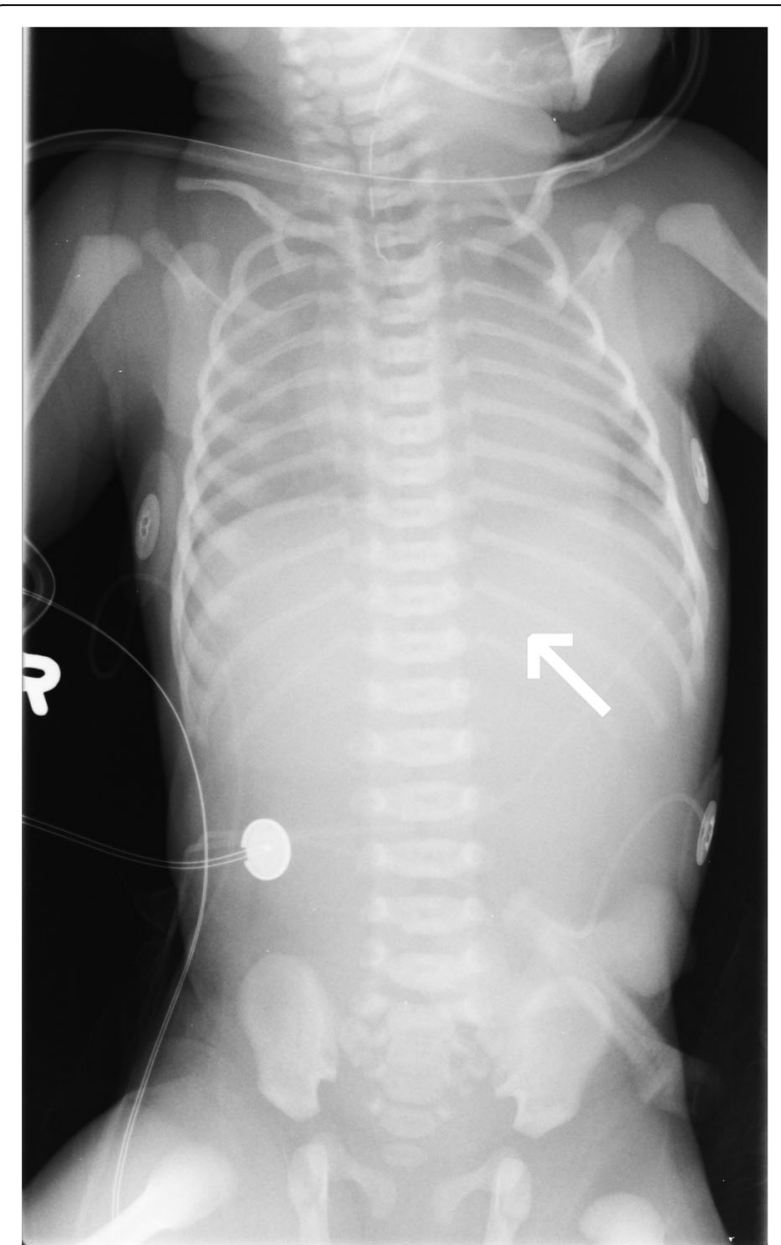

Fig. 23 A 0-day-old female with no gas in the distal bowel (white arrow) suggesting esophageal atresia without

tracheoesophageal fistula

echogenic filling defect within the vein, venous luminal expansion, lack of detectable flow during Doppler evaluation, and inability to compress the vein. Echocardiography can also be used to find clot in the central pulmonary arteries or within the heart [24]. Lung ventilation-perfusion scintigraphy is a safe and sensitive diagnostic tool for PE. Regions that lack radiotracer activity indicates diminished blood flow [24].

$\mathrm{CT}$, especially MDCT, is the imaging modality of choice for PE evaluation. Acute PE is diagnosed when sharply marginated complete or partial pulmonary arterial filling defect, either localized centrally or with an acute margin with the vessel wall, is present on at least two consecutive images (Fig. 21). Chronic PE is usually eccentric and adherent to the vessel wall and may have recanalization of the pulmonary artery [24].

\section{Aortic aneurysms}

Aortic aneurysm is a rare, but important cause of mortality in the pediatric population. Causes can be 


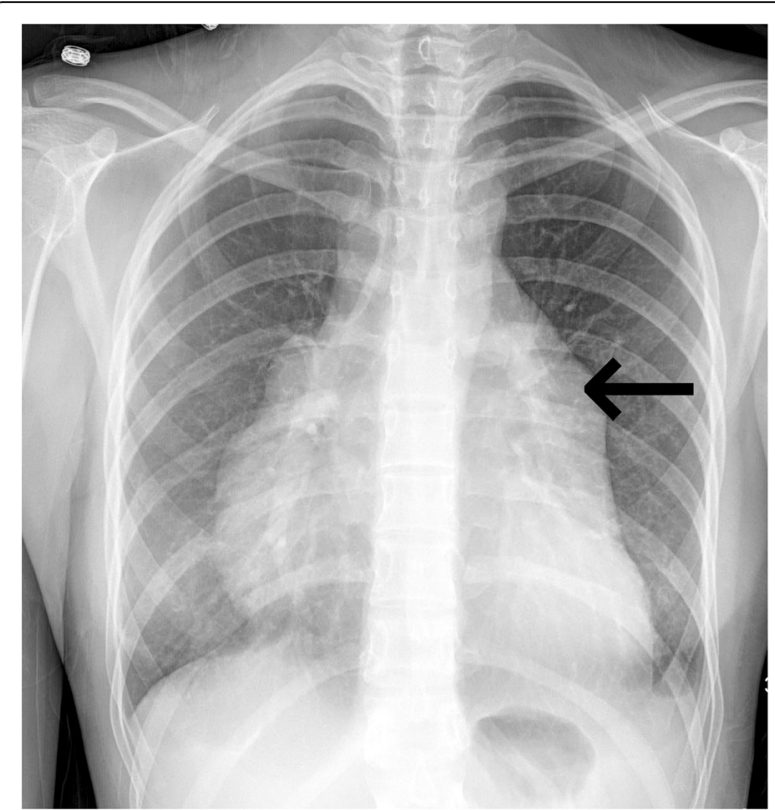

Fig. 24 A 17-year-old female with a history of acute leukemia with an anterior mediastinal mass (black arrow)

non-genetic and genetic in origin. Non-genetic causes include bicuspid aortic valve, coarctation of the aorta, tetralogy of Fallot, and aortitis syndromes [25]. Some of the genetic causes of aortic aneurysms include Marfan syndrome and Ehler-Danlos syndrome. Marfan syndrome is an autosomal dominant inherited syndrome due to mutations in the fibrillin 1 gene on chromosome 15q21. This results in elastic fiber fragmentation and cystic medial necrosis. Cardiovascular manifestations include aortic aneurysms (Fig. 22) and are the most common cause of mortality. Although not always emergent, these aneurysms have a potential to rupture. The aneurysms may occur in the ascending aorta, abdominal aorta, or aortic branches [25]. Ehler-Danlos syndrome is a disorder of collagen and extracellular matrix. Cardiovascular findings, including aortic aneurysms, are seen in the subtype IV, in which collagen type III alpha-1 gene mutation causes an abnormal type III collagen in the vascular wall, skin, and other organs [25]. Comprehensive evaluation with multimodality imaging, including $\mathrm{ECHO}$, multi-slice spiral CT angiography, and magnetic resonance angiography, are recommended to evaluate patients with congenital aortic aneurysms. Management largely follows the literature for adults due to limited studies in congenital aortic aneurysms: conservative management or surgical repair. There is presently no definite size criteria for surgical repair [26]. In the event of a rupture, the patient requires emergent intervention as mortality reaches $80 \%$.

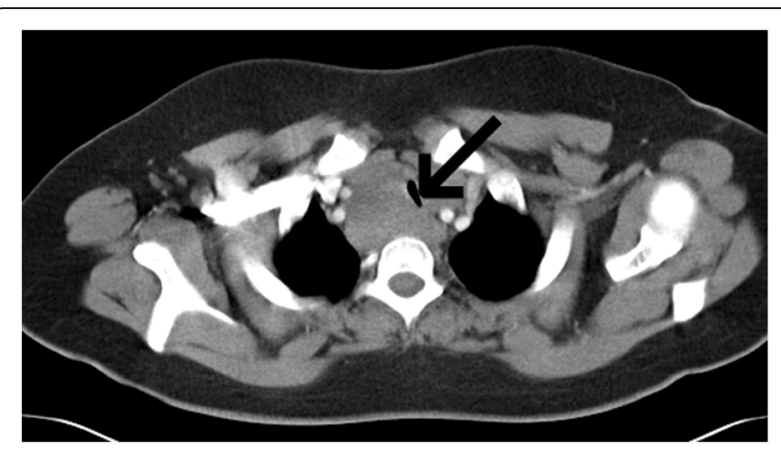

Fig. 25 A 16-year-old female with a history of Hodgkin's lymphoma with severe tracheal narrowing (black arrow)

\section{Esophageal etiologies \\ Esophageal atresia with or without tracheoesophageal fistula}

Esophageal atresia (EA) with or without tracheoesophageal fistula (TEF) occurs in 2 in 10,000 births with male predominance [27]. This is associated with congenital malformations and VACTERL (vertebral, anal, cardiac, tracheal, esophageal, renal, and limb anomalies) or CHARGE (coloboma, heart defects, atresia choanae, growth retardation, genital abnormalities, and ear abnormalities) syndrome. There are five anatomic variations with EA: EA without TEF (Type A), EA with proximal TEF (Type C), EA with distal TEF (Type C), EA with TEF between both esophageal segments and trachea (Type D), and TEF without EA or H-type fistula (Type

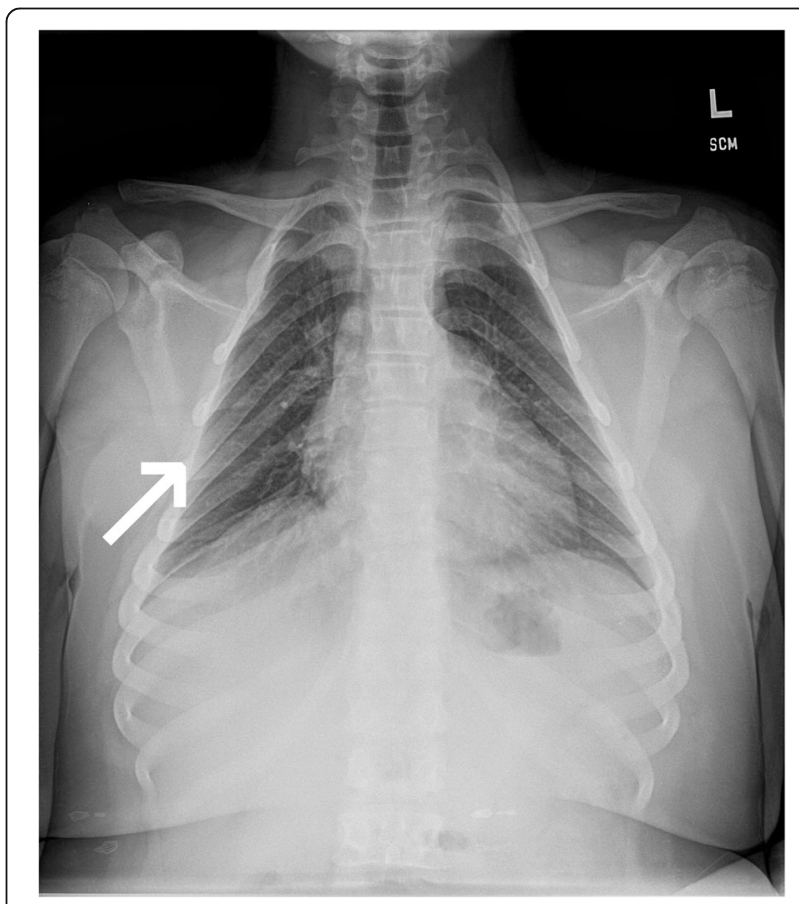

Fig. 26 A 13-year-old male with hypoplastic chest wall (white arrow) demonstrated on plain film 


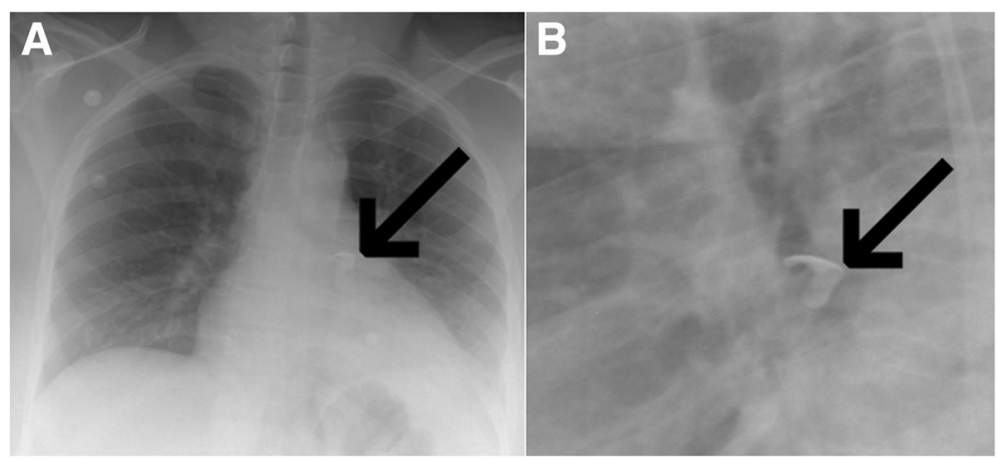

Fig. 27 a, b A 30-year-old female with tooth lodged in the left main bronchus (black arrows) seen on frontal and lateral views

E) [27]. Distal TEF (Type C) is the most common anomaly (85-90\%) [27]. All types except $\mathrm{H}$-type present early in life with respiratory distress and choking. Radiograph should include the abdomen to assess presence of bowel gas. Findings on imaging include gas distended in the gastrointestinal tract (Types $\mathrm{C}$ and $\mathrm{D}$ ), feeding tube that curls at level of atresia if the atresia is associated with TEF [28], or no distal bowel gas if there is no TEF (Types A and B) (Fig. 23). An esophagogram would show distension of the proximal pouch with no air passing distally in the esophagus. Contrast esophagography can provide a definitive diagnosis, while transesophageal ultrasonography, endoscopy, CT, and MRI can provide additional anatomical information of the duplication [27]. EA with or without TEF are usually repaired in the neonatal age. Preoperatively, the airway should be managed to prevent aspiration of gastric contents [27].

\section{Mediastinal etiologies \\ Thoracic tumors}

Tumors can be located in the anterior, middle, or posterior mediastinum and cause narrowing of the trachea or major bronchi. The most common tumors causing large mediastinal masses with respiratory compromise are leukemia and lymphomas (Figs. 24 and 25). It is important to evaluate for potential lifethreatening airway obstruction before using general anesthesia or heavy sedation. The mass effect can be seen with infantile hemangioma, rhabdomyosarcoma, neurogenic tumor, germ cell tumor, and thyroid neoplasm. Tumors that arise from sympathetic tissues, such as neuroblastoma, ganglioneuroblastoma, and ganglioneuroma, can be located in the posterior mediastinum [1]. The degree of cellular maturation can determine the tumor aggressiveness, for example, immature tumors tend to be aggressive, while mature tumors tend to be benign [1]. The standard of care is to manage the airway and to obtain cross-sectional imaging with contrast. MRI and PET-CT can be used to stage and follow-up diseases such as lymphoma.

\section{Pneumomediastinum}

Pneumomediastinum is defined as a mediastinal air leak [29]. Causes of pneumomediastinum vary from alveolar rupture from barotrauma secondary to asthma exacerbation to aspirated foreign bodies, which may be emergent. This is diagnosed with chest radiograph with signs, including the continuous diaphragm sign and linear bands of mediastinal air paralleling the left side of the heart and the descending aorta extending superiorly along the great vessels. The "spinnaker sign" is seen when the thymus is shifted above the heart by pneumomediastinal air [29].

\section{Tracheal obstruction}

Tracheal or laryngeal tumors can manifest as acute upper airway obstruction. Endoluminal masses include recurrent respiratory papillomatosis, laryngoceles, and subglottic hemangiomas. Extraluminal masses include bronchogenic cysts, lymphadenopathy, and neuroblastoma. Radiographs demonstrate narrowing of the trachea [30].

\section{Thoracic cage etiologies}

Chest wall abnormalities, such as hypoplastic thorax, can result in compromised respiratory function. Narrowing of the superior anterior mediastinal space can lead to compression of the mediastinal vessels in airways, resulting in airway obstruction. This is most often seen at the thoracic inlet and left main bronchus. Hypoplastic thorax occurs in skeletal dysplasias, including thanatophoric dysplasia [31]. Thanatophoric dysplasia is the most common lethal skeletal dysplasia caused by mutations of the gene-encoding fibroblast growth factor receptor 3. There are instances of sporadic hypoplastic chest wall which is diagnosed later in life. Chest radiographs provide initial diagnostic information (Fig. 26), while CT will further characterize the pathology with complications. 


\section{Miscellaneous etiologies \\ Foreign body aspiration/ingestion}

Foreign body aspiration is common among young children between 6 months and 3 years of age. Organic materials, such as vegetables, are the most common foreign bodies inhaled. The foreign body is usually inhaled into the main stem bronchi, with right and left bronchi equally involved. Chest radiography with lateral view can assist in localizing the object to the esophagus or airway (Fig. 27a, b). Findings of obstructive emphysema, contralateral mediastinal shift, and ipsilateral restricted diaphragmatic motion during breathing help confirm the diagnosis. Virtual bronchoscopy might be helpful in doubtful cases [1].

\section{Conclusions}

There are various etiologies for non-traumatic thoracic emergencies in the pediatric population. Emergent chest conditions can be related to the lungs, cardiovascular pathologies, and thoracic cage. The timely diagnosis of these conditions is essential for the acute management of these emergencies.

\section{Abbreviations}

ASD: Atrial septal defect; CDH: Congenital diaphragmatic hernia; CLE: Congenital lobar emphysema; CT: Computed tomography; EA: Esophageal atresia; MAS: Meconium aspiration syndrome; MDCT: Multidetector computed tomography; MRI: Magnetic resonance imaging; PE: Pulmonary embolism; PIE: Acute pulmonary interstitial emphysema; RDS: Respiratory distress syndrome; TEF: Tracheoesophageal fistula; VSD: Ventricular septal defect

\section{Acknowledgements}

The authors thank John Pham, MD for the literature search and writing assistance.

\section{Authors' contributions}

$M X W$ and $A B$ contributed in drafting the article and revising it critically for important intellectual content. DR is the guarantor of the integrity of the entire study, study concepts, and design and contributed in drafting the article and revising it critically for important intellectual content. All authors have read and approved the final version of the manuscript.

\section{Funding}

None

\section{Availability of data and materials}

Not applicable

Ethics approval and consent to participate

Not applicable

\section{Consent for publication}

Not applicable

\section{Competing interests}

The authors declare that they have no competing interests.

\section{Author details}

'Department of Radiology, University of Florida College of Medicine, 1600 SW Archer Road, Gainesville, FL 32611, USA. ²Department of Radiology, University of Texas at San Antonio, 7703 Floyd Curl Drive, San Antonio, TX 78229, USA.
Received: 14 June 2019 Accepted: 16 July 2019

Published online: 02 September 2019

\section{References}

1. de Lange C (2011) Radiology in paediatric non-traumatic thoracic emergencies. Insights Imaging 2:585-598. https://doi.org/10.1007/ s13244-011-0113-4

2. Pramanik AK, Rangaswamy N, Gates T (2015) Neonatal respiratory distress. Pediatr Clin North Am 62:453-469. https://doi.org/10.1016/.jpcl.2014.11.008

3. Gereige RS, Laufer PM (2013) Pneumonia. Pediatr Rev 34:438-456. https:// doi.org/10.1542/pir.34-10-438

4. Berrocal T, Madrid C, Novo S, Gutiérrez J, Arjonilla A, Gómez-León N (2004) Congenital anomalies of the tracheobronchial tree, lung, and mediastinum: embryology, radiology, and pathology. RadioGraphics 24:e17-e17. https:// doi.org/10.1148/rg.e17

5. Biyyam DR, Chapman T, Ferguson MR, Deutsch G, Dighe MK (2010) Congenital lung abnormalities: embryologic features, prenatal diagnosis, and postnatal radiologic-pathologic correlation. RadioGraphics 30:1721-1738. https:/doi. org/10.1148/rg.306105508

6. Deshmukh S, Rubesova E, Barth R (2010) MR assessment of normal fetal lung volumes: a literature review. AJR Am J Roentgenol 194:W212-W217. https://doi.org/10.2214/AJR.09.2469

7. Fanaroff AA (2008) Meconium aspiration syndrome: historical aspects. J Perinatol 28:S3-S7. https://doi.org/10.1038/jp.2008.162.

8. Cleveland $\mathrm{RH}$ (1995) A radiologic update on medical diseases of the newborn chest. Pediatr Radiol 25:631-637

9. Agrons GA, Courtney SE, Stocker JT, Markowitz RI (2005) Lung disease in premature neonates: radiologic-pathologic correlation. RadioGraphics 25: 1047-1073. https://doi.org/10.1148/rg.254055019

10. Donnelly LF, Frush DP (1999) Localized radiolucent chest lesions in neonates: causes and differentiation. Am J Roentgenol 172:1651-1658. https://doi.org/10.2214/ajr.172.6.10350310

11. Durell J, Lakhoo K (2014) Congenital cystic lesions of the lung. Early Hum Dev 90:935-939. https://doi.org/10.1016/j.earlhumdev.2014.09.014

12. Dillman JR, Sanchez R, Ladino-Torres MF, Yarram SG, Strouse PJ, Lucaya J (2011) Expanding upon the unilateral hyperlucent hemithorax in children. RadioGraphics 31:723-741. https://doi.org/10.1148/rg.313105132

13. Taylor GA, Atalabi OM, Estroff JA (2009) Imaging of congenital diaphragmatic hernias. Pediatr Radiol 39:1-16. https://doi.org/10.1007/s0024 7-008-0917-7

14. Liu AH, Covar RA (2008) Asthma. In: Taussig L, Landau L (eds) Pediatr. Respir. Med, 2nd edn. Mosby Elsevier, Philadelphia, p 806

15. Halaby C, Feuerman M, Barlev D, Pirzada M (2014) Chest radiography in supporting the diagnosis of asthma in children with persistent cough. Postgrad Med 126:117-122. https://doi.org/10.3810/pgm.2014.03.2747

16. Wang ZJ, Reddy GP, Gotway MB, Yeh BM, Higgins CB (2003) Cardiovascular shunts: MR imaging evaluation. radiographics 23:S181-S194. https://doi. org/10.1148/rg.23si035503

17. Penny DJ, Vick GW (2011) Ventricular septal defect. Lancet (London, England) 377:1103-1112. https://doi.org/10.1016/S0140-6736(10)61339-6

18. Abdulla R, Luxenberg DM (2011) Cardiac interpretation of pediatric chest Xray. Hear. Dis. Child. Springer US, Boston, pp 17-34. https://doi.org/10.1007/ 978-1-4419-7994-0 2

19. Goo HW, Park I-S, Ko JK, Kim YH, Seo D-M, Yun T-J et al (2003) CT of congenital heart disease: normal anatomy and typical pathologic conditions. RadioGraphics 23:S147-S165. https://doi.org/10.1148/rg.23 si035501

20. Imazio M, Adler Y (2013) Management of pericardial effusion. Eur Heart J 34 1186-1197. https://doi.org/10.1093/eurheartj/ehs372

21. Blanco CC, Parekh JB, Adam HM (2010) Pericarditis. Pediatr Rev 31:83-84. https://doi.org/10.1542/pir.31-2-83

22. Pérez-Casares A, Cesar S, Brunet-Garcia L, Sanchez-de-Toledo J (2017) Echocardiographic evaluation of pericardial effusion and cardiac tamponade. Front Pediatr 5:79. https://doi.org/10.3389/fped.2017.00079

23. Buck JR, Connors RH, Coon WW, Weintraub WH, Wesley JR, Coran AG (1981) Pulmonary embolism in children. J Pediatr Surg 16:385-391. https://doi. org/10.1016/S0022-3468(81)80700-2

24. Thacker PG, Lee EY (2015) Pulmonary embolism in children. AJR Am J Roentgenol 204:1278-1288. https://doi.org/10.2214/AJR.14.13869

25. Karakurt C (2013) Aortic aneurysm in children and adolescents. Aortic Aneurysm - Recent Adv, InTech. https://doi.org/10.5772/53383 
26. Wang Y, Tao Y (2015) Diagnosis and treatment of congenital abdominal aortic aneurysm: a systematic review of reported cases. Orphanet I Rare Dis 10:4. https://doi.org/10.1186/s13023-015-0225-x

27. Achildi $\mathrm{O}$, Grewal $\mathrm{H}$ (2007) Congenital anomalies of the esophagus. Otolaryngol Clin North Am 40:219-244. https://doi.org/10.1016/j.otc.2 006.10 .010

28. Berrocal T, Torres I, Gutiérrez J, Prieto C, del Hoyo ML, Lamas M (1999) Congenital anomalies of the upper gastrointestinal tract. RadioGraphics 19: 855-872. https://doi.org/10.1148/radiographics.19.4.g99j05855

29. Hauri-Hohl A, Baenziger O, Frey B (2008) Pneumomediastinum in the neonatal and paediatric intensive care unit. Eur J Pediatr 167:415-418. https://doi.org/10.1007/s00431-007-0517-9

30. Darras KE, Roston AT, Yewchuk LK (2015) Imaging acute airway obstruction in infants and children. radiographics 35:2064-2079. https://doi.org/10.1148/ rg.2015150096

31. Dighe M, Fligner C, Cheng E, Warren B, Dubinsky T (2008) Fetal skeletal dysplasia: an approach to diagnosis with illustrative cases. RadioGraphics 28: 1061-1077. https://doi.org/10.1148/rg.284075122

\section{Publisher's Note}

Springer Nature remains neutral with regard to jurisdictional claims in published maps and institutional affiliations.

\section{Submit your manuscript to a SpringerOpen ${ }^{\circ}$ journal and benefit from:}

- Convenient online submission

- Rigorous peer review

- Open access: articles freely available online

- High visibility within the field

- Retaining the copyright to your article 